\title{
POST-OPERATIVE RETENTION OF URINE
}

\author{
By BERNARD H. HAND, F.R.C.S. \\ Dspt. of Surgical Studies, Middlesex Hospital
}

\section{Introduction}

Post-operative retention of urine is a common surgical complication, and it is essential to have a clear understanding of the factors involved in its production and the principles of its management, if further complications and mortality are to be prevented.

The object of this article is not only to describe the methods of prevention and relief of urinary retention but to point out when they are indicated. The post-operative treatment of cases who have undergone surgery to the bladder, prostate and urethra will not be considered.

\section{Diagnosis}

While in most cases the diagnosis of retention of urine presents no problem, a word of warning is needed, however, in two types of case.

First, in the immediate post-operative period it is usual for less urine to be secreted, the degree of oliguria being proportional to the severity of the operation, and sometimes anuria may occur from renal damage. Either of those conditions would account for the patient's failure to pass water. It is therefore necessary to palpate the abdomen and confirm the presence of a distended bladder before assuming that urinary retention has occurred. If no urine has been passed within 24 hours of operation and the bladder is not palpable it is necessary to pass a catheter to determine the quantity and character of what little urine has been secreted.

Secondly, elderly and middle-aged male patients even with retention of urine may make no complaint because they are passing water and have little or no pain-they may even complain of frequency of micturition. However, the volume of urine being passed is only $\mathrm{I}-3$ ounces at a time and palpation of the abdomen will reveal a distended bladder. This is a comparable situation to the patient who is passing frequent small loose motions when in fact the rectum is obstructed by a mass of hard impacted faeces.

\section{Treatment \\ Prevention}

While it is not possible to eliminate the condition, unquestionably much can be done to reduce its incidence in all types of case. The following measures are therefore stressed:

I. A few words of encouragement are useful, particularly to those patients who report that they could not pass water after a previous operation.

2. As many patients experience difficulty in passing water while in bed, it gives them great confidence post-operatively if they practice this before the operation, and there is no reason why this should not be advised as a routine in all routine cases.

3. It is important that all patients go to the operating theatre with an empty bladder. While it may be necessary for technical reasons, in addition it allows a longer period of time to elapse before the bladder is full again, during which the patient is able to recover his faculties and become aware of the situation before overdistention develops. This is clearly a nursing problem and its importance and rationale should be stressed upon the nursing staff.

4. The more distended the bladder becomes the more difficult it is to micturate; patients should therefore be asked to try and pass water as soon after operation as practical. Again, this is a nursing problem and some routine should be adopted whereby the house surgeon is informed if a patient has not been successful within some stipulated time.

5. During the course of taking a full history, and performing a full clinical examination, it is important to assess the patient's ability to micturate, particularly in middle-aged and elderly males. It may be that the degree of prostatic obstruction which already exists is such that post-operative retention is almost inevitable. Further, patients are sometimes admitted for surgery, particularly in an emergency, with chronic 
urinary retention from obstruction, in addition to the primary condition. In these cases careful consideration has to be given to decide which condition takes priority. In reaching this decision the following factors must be taken into account:

(a) The age and general condition of the patient, and in particular his ability to survive two anaesthetics and two operations; the opinion of the anaesthetist should be sought on this point.

(b) The cause and degree of obstruction and its rate of progression are more important than the size of the prostate as felt on rectal examination.

(c) The nature and urgency of the proposed operation must be taken into account. Clearly in the case of a strangulated hernia, or emergency gastrectomy, no delay is possible.

(d) If time permits a full investigation by means of bacteriological examination of the urine, blood urea, intravenous pyelogram should be carried out, to show the state of renal function and the degree of chronic retention. Cystoscopy should be avoided owing to the danger of inducing retention, infection or haemorrhage, unless the surgeon is prepared to proceed immediately to remove the obstruction.

In the light of the information obtained, the following alternatives are possible:

(i) The primary condition is treated, risking the development of urinary retention.

(ii) The obstruction is dealt with in the first instance and after a suitable interval the primary condition is treated.

(iii) Where it is not essential to treat the primary condition at all, advise against surgery of any kind. For instance, an elderly man with symptoms of urinary obstruction should have an uncomplicated inguinal hernia controlled by a truss, rather than risk the possibility of two operations.

6. In certain operations experience has shown that even without any degree of pre-operative urinary obstruction, post-operative retention of urine is extremely likely. In these cases it is usual for a self-retaining urethral catheter to be inserted before, or immediately after, operation and left in place for several days. Thus it has become a routine after anterior perineal repairs, operations for stress incontinence, Wertheim's hysterectomy and abdomino-perineal resection of the rectum.

\section{Treatment of the Established Condition}

For purposes of description it is deemed advisable to divide the cases into three groups, defining each group and considering its management separately.

\section{Group I}

In these patients no organic obstruction exists pre-operatively and none is induced by surgery. Here psychological and postural factors are $z$ responsible and treatment presents little difficulty. $\stackrel{\mathbb{Q}}{\complement}$

The solution to the problem should not be $c$. simply to pass a catheter. While little harm will $\overrightarrow{\vec{s}}$ result, if properly carried out, in this group simpler measures should be tried first and are frequently successful.

(I) Simple Measures. A short talk with the $\frac{\overline{\frac{\rho}{\sigma}}}{\bar{\phi}}$ patient, explaining that nothing has been done $\stackrel{\square}{\circ}$ operatively to interfere with his ability to mic- क turate, will help to reassure him. It is valuable $\overrightarrow{0}$ to point out that overstraining will not help, and that initiation of micturition is achieved by $\vec{\omega}$ relaxation of the perineal musculature. Patients $\frac{\sigma}{0}$ are sometimes assisted by putting screens round $\frac{0}{3}$ the bed, placing a hot-water bottle on the hypo- $\frac{3}{\omega}$. gastrium, using a warmed bedpan or urinal, $\omega$ turning on taps and sitting over the edge of the $\omega_{\infty}$ bed. If the patient's general condition and the operative procedure permits, he should be stood 워 out of bed, supported if necessary by a nurse or 0 house surgeon, preferably of male sex. A small enema and removal of a rectal tube inserted at $\vec{\nabla}$ operation are sometimes helpful. In an agitated $\underset{\varnothing}{\mathbb{D}}$ patient a small dose of morphia may be given.

(2) Carbachol. If the above measures fail Ca民 bachol (carbamyl choline chloride) may be eng $\frac{\Phi}{-}$ ployed. To be effective $0.25 \mathrm{mg}$. should $\overrightarrow{0}$ given subcutaneously or intramuscularly, and the dose may be repeated half an hour later. If this is not successful no more should be given. Since Carbachol acts on most smooth muscle, some surgeons do not permit its use in cases where a gastro-intestinal anastomosis has been performed, in case a violent wave of peristalsis is produced and the anastomosis damaged. It is contraindicated in cardiac and shocked cases since it may cause a fall in blood pressure with peripheral vasodilation. Some patients appear hypersensitive and may sweat, feel nauseated and faint. Atropine may be used as an antidote. While Carbachol requires to be given parenterally for 3 . acute retention, should the bladder be hypotonic after retention is relieved, oral Carbachol $2 \mathrm{mg}$. 궁 three times a day does help to improve the o bladder tone.

(3) Urethral Catheterization. If simple man- $\frac{D}{0}$ oeuvres and injection of Carbachol have failed catheterization should now be performed. This $\mathcal{N}$ must be carried out with the utmost gentleness N and scrupulous aseptic technique. There is much $\mathrm{C}_{\mathrm{W}}$ to be said in favour of performing this in the $\omega$ operating theatre, but clearly in a busy general hospital this may be impractical-but a mask and rubber gloves should be worn. Scrubbing up should be performed after the external genitals have been swabbed. A soft rubber catheter 
size 16-18 (French) of the Jacques or Harris type, well lubricated with sterile jelly or liquid paraffin, is used. Some advise that a local anaesthetic, such as I : $I, 500$ nupercaine, should be used. While not essential it has the advantage that, after instillation, the patient may micturate spontaneously. The catheter should not be touched with the hand but held with sterile gauze. Subsequently, the patient should be encouraged to drink freely, if his operation permits, and attempt to pass water at frequent intervals thereafter. With the above technique in this group it should not be necessary to prescribe any chemotherapy.

\section{Group 2}

No organic obstruction exists pre-operatively, but the operation leads to its development. Usually the obstruction is of a temporary nature and resolves within a few days, being due to traumatic oedema or damage to the pelvic autonomic nerves. Here treatment only occasionally presents a problem. Many of this group fall into the category in which an indwelling catheter is employed since difficulty is anticipated. If not, then the procedures mentioned under group $I$ should be followed, and if a catheter is passed it may be advisable to leave it in situ for a few days draining the bladder intermittently rather than continuously to allow recovery of tone. The catheter can be removed when abdominal distension has been relieved, or if the patient passes urine round the catheter or becomes aware of bladder distension.

\section{Group 3}

Some degree of obstruction exists pre-operatively which is rendered complete by a combination of the factors at play in groups $I$ and 2 . This group frequently poses difficult problems in management and increases the morbidity and mortality of surgery. The conditions which are concerned here are bladder-neck obstruction from benign hypertrophy, carcinoma, fibrous or calculus disease of the prostate, urethral stricture, and finally neurological diseases such as tabes dorsalis and disseminated sclerosis.

\section{Bladder-neck Obstruction}

In this condition there are two types of case to be considered, the first developing acute retention where no previous chronic retention existed pre-operatively, and the second where acute retention is superimposed on a pre-existing chronic retention. The latter should be detected in the pre-operative phase of a routine case and the cause treated primarily-but in an emergency, even if detected it would take second place to the treatment of the primary disease. The distinction between these groups can be made by a consideration of the past history of urinary symptoms and the height of the bladder above the pubis. Obviously these two types are not absolutely clear-cut entities, but when faced with borderline cases it is important to decide from the beginning which line to adopt so that suprapubic catheterization or cystotomy do not require to be carried out after several urethral catheterizations have failed to establish micturition.

In the first type conservative measures should certainly be attempted as they are sometimes successful. Some advise that Carbachol should be administered; but since it is unlikely to be successful and theoretically could cause rupture of a diseased bladder, its use is perhaps unwise. One of the following measures should now be adopted:

(a) Urethral catheterization.

(b) Perineal urethrostomy.

(c) Suprapubic aspiration.

(d) Suprapubic catheterization.

(e) Suprapubic cystotomy.

In the other type, with acute on chronic retention, if simple measures have failed, urethral catheterization and suprapubic aspiration should not be employed since they are unlikely to succeed in establishing micturition where the obstruction was so well developed pre-operatively. Either suprapubic catheterization or cystotomy are therefore required with slow decompression of the bladder over a period of 24 hours.

(a) Urethral Catheterization. In cases where the pre-operative obstruction is not marked, urethral catheterization may be successful in establishing spontaneous micturition. If performed, the aseptic and gentle technique is particularly important in these cases since infection is more likely to occur and in association with obstruction will be persistent until the source of obstruction is removed. A soft rubber catheter may easily fail to pass the obstruction and rather than pass two catheters a bicoudé gum elastic catheter or Tiemann's rubber catheter, which has a solid curved tip, should be employed in the first instance. The danger of urethral catheterization in these cases is that, however gently performed, the superadded trauma is likely to increase the oedema and mitigate against the patient passing water of his own accord later. A further danger, with a vascular prostate, is haemorrhage and conversion of the case from one of urinary retention to one of clot retention with all its hazards. After catheterization it is inadvisable to leave the catheter in situ as this increases the chance of oedema and infection; also a limit must be put on the number of times it may be repeated, 
depending upon the circumstances. If catheterization has proved successful a short course of chemotherapy or Haustus hexamine compositus is advisable. The latter renders the urine acid, reduces infection, and by its mildly irritant effect often assists micturition.

(b) Perineal Urethrostomy. Namely, the insertion of a self-retaining catheter via the perineal urethra, has been advised in certain cases of urinary retention by Sandrey (1949), and may be employed in the post-operative period. Although it spares the anterior urethra, ascending infection is not prevented and the method has not been generally accepted.

(c) Suprapubic Aspiration. This method, although not in common use, has definite advantages over urethral catheterization. First, as nothing passes through the urethra, infection is less likely to occur; secondly, the oedema of the bladder neck is not increased by the trauma of the procedure, and the patient is therefore more likely to pass water spontaneously afterwards; thirdly, prostatic haemorrhage does not occur; fourthly, it is a simple procedure requiring no special surgical instruments or experience; and lastly, if it fails it does not interfere with any subsequent treatment. While it is not suggested that this method should be used in all cases where urethral catheterization is indicated, it has a real place particularly in the older age groups. It has been employed with success on numerous occasions on this unit.

It can be performed quite easily in the patient's bed with its foot on blocks. The technique employed is as follows: After shaving the pubic hair and painting the skin with iodine, the skin and abdominal wall above the pubis are infiltrated with local anaesthetic. A sterile narrow lumbar puncture needle with a syringe attached is inserted just above the pulsic symphysis in a backward and slightly downwari direction until the bladder is reached. By aspirating as much as possible the bladder can be al most completely emptied. The disadvantages are that it can only be performed at the most three: times; if attempted more frequently extravasation of urine into the cave of Retzius is liable to occur, and further, it is dangerous to attempt it if a scar is present in the lower abdominal wall, in case loops of intestine are adherent to it ; under surface.

(d) Suprapubic Catheterization. Essentially this is a method of suprapubic cystotomy and the technique advised is that described by Riches (1943). It was originally designed for the treatment of paraplegics, but soon became applied to cases of prostatic obstruction.

It is indicated in all cases where the alternative is suprapubic cystotomy, namely, where the above methods have failed in acute retention and all cases where an acute retention is superimposed on $\frac{\varrho}{z}$ a chronic one. It involves the blind introduction $\mathbb{\Phi}$ under local anaesthesia of a modified Malecot $\bar{c}$ catheter (No. I6-I8 French) stretched over an introducer which has a cutting terminal end. The catheter is fixed on the stretch and is held in position distally by a hinged arm, which is con- $\underline{\underline{O}}$ trolled proximally. The orifice made in the $\frac{\bar{\omega}}{\vec{\sigma}}$ bladder is smaller than the unstretched catheter $\stackrel{\mathbb{\Phi}}{\varrho}$ and the tract oblique, thereby reducing the danger $\cong$ of leakage and permitting rapid closure when the $\vec{s}$ obstruction has been removed. Space does not permit a full description of the technique and $\vec{\omega}$ readers are referred to the original descriptions by $\stackrel{\sigma}{\circ}$ Riches; however, a few points are stressed. It $\mathbb{C}$ should always be performed in the operating theatre with the table tilted into a slight Tren- $\omega$ delenburg position. The catheter should only $\omega$ be inserted blindly if the dome of the bladder $\infty$ can be definitely felt. If not, then surface of the bladder should be exposed through a small incision and insertion performed under direct vision. $\frac{\text { ? }}{3}$ The site of insertion should be midway between the pubic symphysis and the top of the bladder, $\mathbb{D}$ but never higher than midway between the pubic $\overparen{\Phi}$ symphysis and the umbilicus. The catheter and 3 introducer should be well lubricated. An incision about $\frac{1}{4}$ in. long is made in the anterior abdomina $\vec{A}$ wall and the linea alba. After insertion it is vitu to fix the catheter and shield firmly to the abdof minal wall, since, should it come out within the first seven days it may be difficult or impossible to replace it, particularly if not attempted immediately.

The disadvantage of the method is that it $\stackrel{\circ}{\circledR}$ requires two special instruments and a knowledge $\overrightarrow{\overrightarrow{0}}$ of the technique which may not be available in 3 an emergency. It is under these circumstances that suprapubic cystotomy is indicated.

(e) Suprapubic Cystotomy. The technique is as follows: A local or general anaesthetic may be employed, with the patient in a slight Trendelen- $\frac{5}{3}$ burg position a short median sub-umbilical incision is made, passing between the recti muscles. $\frac{\rho}{3}$ The peritoneum is identified and retracted upwards and the dome of the bladder identified, two 은 stay sutures are inserted and either a Morson's $D$ or Kidd's bladder trocar and canula inserted into the bladder. A self-retaining catheter of a N Malecot or De Pezzer type is inserted, taking care not to spill urine into the wound, and tied in $N$ with catgut. The wound is closed in layers with $\underset{\omega}{ }$ a drain in the cave of Retzius in case of urinary leakage.

Emergency Prostatectomy

In recent years emergency prostatectomy has 
found a definite place in the treatment of urinary retention due to benign prostatic enlargement, in cases where renal function is considered adequate and infection is either absent or minimal. It is too early for it to have found a place in cases where the retention has presented as a postoperative manifestation. However, the time may come when such a place is found provided the above criteria are fulfilled, the general condition of the patient permits it and the two operative fields do not transgress one another.

\section{Urethral Stricture}

Any patient with a severe stricture - of the urethra is likely to report this pre-operatively, so we need only consider a passable stricture which has become further narrowed by oedema. Simple measures should be tried first, and while Carbachol is unlikely to do harm it is unlikely to be successful and is best avoided in these cases. Dilatation of the structure is therefore required. This can be performed under local anaesthesia with full aseptic precautions. A filiform gum elastic or plastic bougie is passed to the face of the stricture. It should possess a thread at the proximal end to allow follow-through bougies of a larger size to be attached. One bougie may not negotiate the orifice and it is then necessary to pass several of the same type, leaving them all in the urethra -and one can usually be passed through the stricture (faggot method). The oth ers are then removed and $a$ gradual dilatation $p$ erformed by attaching larger sizes in turn. The bladder is then emptied. Under these circum stances dilatation should only be carried out $f$ ar enough to permit micturition, full treatment bein $g$ postponed till later in the post-operative period.

Occasionally general anaesthesia is required to achieve the dilatation. In either case it is advisable to prescribe a short course of chemotherapy.

\section{The Neurological Bladder}

Should a tabetic or a patient with disseminated sclerosis develop post-operative retention, the advice of a neurologist should be obtained before any suprapubic drainage is instituted; since closure of the fistula may never be possible and the patient is left with a permanent suprapubic tube. Urethral catheterization is therefore indicated in the first instance.

\section{BIBLIOGRAPHY}

RICHES, E. W. (1943), Lancet, ii, 128.

RICHES, E. W. (1950), Brit. Surg. Prac., 7, 158.

SANDREY, J. G. (1949), Postgrad. med. F., 25, 71 .

\section{ANAESTHETICS (Special number of Postgraduate Medical Journal, September 1955)}

Price: 3s. 9d. post free

THE MODERN VIEW OF ANAESTHESIA

G. S. W. Organe, M.D., D.A., F.F.A.R.C.S. THE PRODUCTION OF UNCONSCIOUSNESS

B. G. B. Lucas, D.A., F.F.A.R.C.S.

ANALGESIA

J. B. Wyman, M.B.E., D.A., F.F.A.R.C.S. MUSCLE RELAXATION IN SURGERY

Angus Smith, F.F.A.R.C.S.

CONTROL OF THE BLOOD PRESSURE AND CONTROLLED HYPOTENSION C. F. Scurr, M.V.O., D.A., F.F.A.R.C.S. CHLORPROMAZINE AND ALLIED
SUBSTANCES

John Beard, M.D., D.A., F.F.A.R.C.S., D.C.H.

CONTROLLED HYPOTHERMIA

E. J. Delorme, M.D., F.R.C.S.(C.)

MANAGEMENT OF THE APNOEIC PATIENT

Ronald Woolmer, D.A., F.F.A.R.C.S.

THE USE OF ANTIDOTES IN ANAESTHESIA

B. A. Sellick, D.A., F.F.A.R.C.S.

Published by

THE FELLOWSHIP OF POSTGRADUATE MEDICINE

60, Portland Place, London, W.1 